\title{
Size effect on the phonon heat conduction in semiconductor nanostructures
}

\section{Abdurrahman Khaleel Suleiman Abdul-Ghefar Kamil Faiq \\ Department of Physics / College of science \\ University of Salahaddin}

Received
$08 / 03 / 2009$
Accepted

05 / 05 / 2009

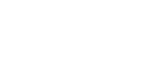

تمت درلسة التوصل الحراري الشبيكي ظاريا فيسليكون (نانو ولير ) و(بئر كمي)

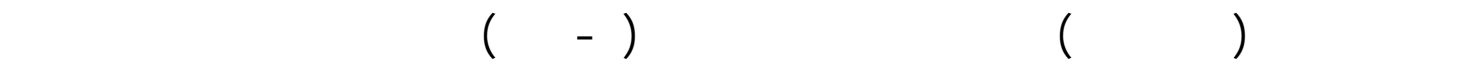

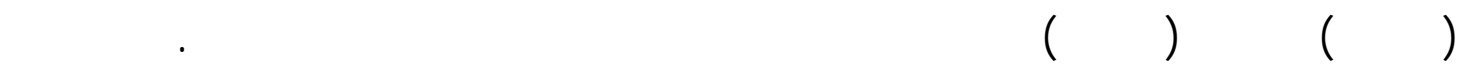

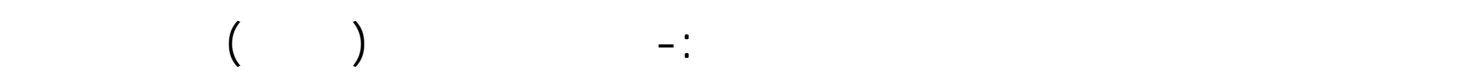
فرق الكتلة، ولسطارة الحدود عند ( . . آ) كلفن.

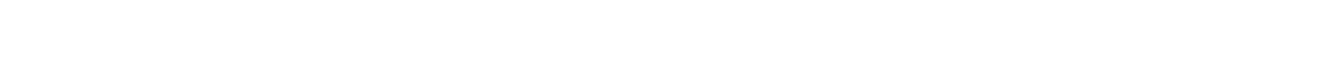

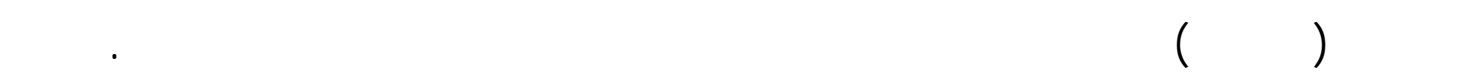

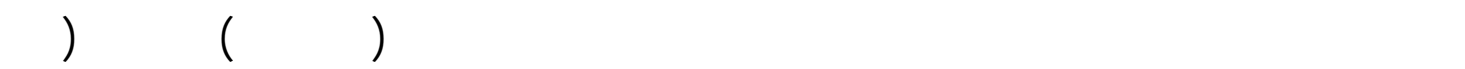

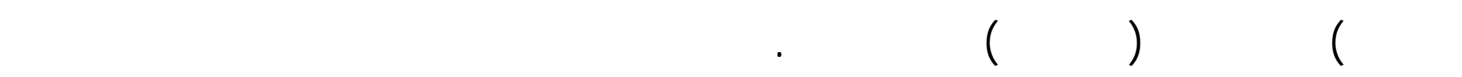
نتائج الأبحك المنشورة وتبين ظابقها فع النتائج العملية كما هي الحل مع النتائج الظرية.

\section{Abstract}

Lattice thermal conductivity for silicon nanowires and quantum well are theoretically investigated in the temperature range from $2 \mathrm{~K}$ to $300 \mathrm{~K}$. The modified Gallaway method for bulk crystal is used for calculating lattice thermal conductivity. All important phonon relaxation mechanism such as Umklapp scattering, Mass-difference scattering and boundary scattering are calculated at $300 \mathrm{~K}$. The result show that the modification of the acoustic phonon modes and phonon group velocities due to spatial confinement of phonons lead to significant increase in the all phonon relaxation rate. From our numerical results, we predicate a significant decrease of the lattice thermal conductivity in cylindrical 
nanowires with diameter $(\mathrm{D}=10-\mathrm{nm})$, and quantum well with thickness of the same size, results compared to that of the reported experimental as well as theoretical values.

\section{Introduction}

The physical properties of nanostructure have been investigated extensively both theoretically and experimentally due to their scientific and industrial importance. Single crystal silicon thin films and layers with thickness in the range of $1 \mathrm{~nm}-100 \mu \mathrm{m}$ are widely used in modern applications such as SOI (silicon-on-insulter) device, integrated circuit transistor, and nanowires applications in the area of IR detectors and IR night vision, and thermal sensor. (Makdadi et al., 2005; Feng et al., 2003).

As the size of low-dimensional materials decreases to the nanometers size range, the thermal, electronic, magnetic, optical, and thermodynamical properties of the material are significantly altered from those of either the bulk or a single molecule. (Feng et al., 2003)

Additionally a large degree of solid-state material behavior depends on phonon dynamics, and these dynamics are substantially altered as nanostructure dimensions approach the phonon mean free-path length. The continuous scaling down of feature sizes in Micro-electronic and Micro-mechanical devices to nanometer sizes leads to increased power dissipation per unit area. This makes it important to understand heat conduction in various kinds of nanostructure, such as: quantum well (two dimension (2D), nanowires and nanotube(1D) and quantum dot(0D), and in particular to understand the effects of the confinement of phonons as the nanostructures size approaches the phonon mean-free-path length. Indeed, in bulk materials internal scattering dominates heat transfer processes. (Alassafee., 2005; Yang et al., 2006; Makdadi et al., 2005)

For crystalline nanowires, though, as its size decreases the frequency of phonon-boundary collisions increases. In addition the ratio of the surface area to volume increases, the result is that the thermal conductivity of nanostructures differs significantly from that of bulk materials this has been shown experimentally by many author's. (Zou et al., 2000; Liang et al., 2006).

In this work the dependence of thermal conductivity on the diameter of free standing nanowires and on the thickness of free standing quantum well is investigated theoretically taking into account spatial confinement of phonon induced by the boundaries which leads to altering phonon spectra.

Our recent work has focused on silicon because:-

1) the material properties are well known.

2) The relatively high sound velocity makes it easier to observe size effect at large diameter and higher temperatures, 3- Experimental measurements are currently available for silicon nanowires and quantum well. 


\section{THEORY}

\section{1- phonon Boltzmann equation:-}

A phonon of energy $\hbar \omega_{S}(q)$ and velocity $V_{S}(q)$ in the direction of $q$ contributes $\hbar \omega_{S}(q) V_{S}(q)$ to the heat current where $q$ represent phonon wave vector. The net phonon heat current with a small temperature gradient $\nabla \mathrm{T}$ is given by:-

$\mathrm{J}_{\mathrm{Q}}=-\Sigma \tilde{\mathrm{N}}_{\mathrm{q} \cdot \mathrm{s}} \hbar \omega_{\mathrm{S}}(\mathrm{q}) \mathrm{V}_{\mathrm{S}}(\mathrm{q})$

Where subscript ${ }_{s}$ refers to a particular phonon polarization type, $\mathrm{V}_{\mathrm{S}}(\mathrm{q})$ is the phonon group velocity, and $\tilde{\mathrm{N}}_{\mathrm{q} . \mathrm{s}}=\mathrm{N}_{\mathrm{q} . \mathrm{s}}^{\mathrm{o}}-\mathrm{N}_{\mathrm{q} . \mathrm{s}}$ is the deviation of the phonon distribution, $\mathrm{N}_{\mathrm{q} . \mathrm{s}}$, from its equilibrium value, $\mathrm{N}_{\mathrm{q} . \mathrm{s}}^{\mathrm{o}}$. The equilibrium phonon distribution, $\mathrm{N}_{\mathrm{q}}^{\mathrm{o}}$ is given by the Bose-Einstein distribution:-

$$
N_{q, s}^{\circ}=\frac{1}{e^{\left(\hbar \omega_{s}(q) / K_{b} T\right)}-1}
$$

By definition

$\mathrm{J}_{\mathrm{Q}}=-\mathrm{K} \nabla \mathrm{T}$

Thus the problem of determining the lattice thermal conductivity is essentially that of obtaining $\tilde{\mathrm{N}}_{\mathrm{q} . \mathrm{s}}$. In order to do this we need to solve the Boltzmann equation for $\tilde{\mathrm{N}}_{\mathrm{q} . \mathrm{s}}$, (Zou et al., 2000; Callawy., 1958). In steady state, the phonon Boltzmann equation can be written as:-

$\left(\frac{\partial N_{q, s}}{\partial t}\right)_{\text {drift }}+\left(\frac{\partial N_{q, s}}{\partial t}\right)_{\text {scatt }}=0$

In equation(4), $\left(\partial \mathrm{N}_{\mathrm{q} . \mathrm{S}} \partial t\right)_{\text {drif }}$ represent the change of the phonon distribution in the presence of a temperature gradient, and it is given by:$\left(\frac{\partial N_{q, s}}{\partial t}\right)_{d r i f t}=-V_{s}(q) \cdot \nabla N_{q, s}=-\left(V_{s}(q) \cdot \nabla T\right) \frac{\partial N_{q, s}}{\partial T}$

The value of $\mathrm{N}_{\mathrm{q} . \mathrm{s}}$ may also change due to scattering by other phonons, impurities, charge carriers, interfaces, boundaries, etc. The change in $\mathrm{N}_{\mathrm{q} . \mathrm{s}}$ due to these process is denoted in eq(4) by $\left(\partial \mathrm{N}_{\mathrm{q} . \mathrm{s}} \partial t\right)_{\text {scatt. }}$ In the relaxation-time approximation this can be written as:-

$$
\left(\frac{\partial N_{q, s}}{\partial t}\right)_{\text {scatt }}=\frac{N_{q, s}^{\circ}-N_{q, s}}{\tau_{C s}(q)}
$$

Where $\tau_{\mathrm{Cs}(\mathrm{q})}$ is the combined phonon relaxation time. Substituting eq(6) and eq(5) in eq(4), we can rewrite the phonon Boltzmann equation as:-

$-V_{x} \frac{\partial N_{q, s}}{\partial x}-V_{y} \frac{\partial N_{q, s}}{\partial y}-V_{z} \frac{\partial N_{q, s}}{\partial z}+\frac{\widetilde{N}_{q, s}}{\tau_{C s}(q)}=0$

Where $\mathrm{V}_{\mathrm{x}}, \mathrm{V}_{\mathrm{y}}$, and $\mathrm{V}_{\mathrm{z}}$ are the three components of phonon group velocity along the $\mathrm{x}, \mathrm{y}$, and $\mathrm{z}$ axis, respectively. Theoretical analysis, and consider free standing cylindrical nanowires and quantum well have the same size $\mathrm{D}$, with an axis along the $\mathrm{z}$ direction, and assume a temperature gradient along this axis, eq(7) then becomes:- 
$V_{x} \frac{\partial \widetilde{N}_{q, s}}{\partial x}+V_{y} \frac{\partial \widetilde{N}_{q, s}}{\partial y}+\frac{\widetilde{N}_{q, s}}{\tau_{C s}(q)}=V_{z} \frac{\partial T}{\partial z} \frac{\partial N_{q, s}}{\partial T}$

Assuming that the phonon distribution does not deviate strongly from its equilibrium value due to the temperature gradient, $\left(\partial \mathrm{N}_{\mathrm{q} . \mathrm{s}} / \partial \mathrm{T}\right)$ replace by $\left(\partial \mathrm{N}_{\mathrm{q} . \mathrm{s}}^{\mathrm{o}} / \partial \mathrm{T}\right)$ in the right-hand side of the eq.(8). This is a standard step used for solving such an equation (Zou et al., (2000; Callawy., 1958). Correspondingly, the linearized phonon Boltzmann equation takes the form:-

$V_{x} \frac{\partial \widetilde{N}_{q, s}}{\partial x}+V_{y} \frac{\partial \widetilde{N}_{q, s}}{\partial y}+\frac{\widetilde{N}_{q, s}}{\tau_{C s}(q)}=V_{z} \frac{\partial T}{\partial z} \frac{\partial N_{q, s}^{\circ}}{\partial T}$

\section{2- Calculation of the Lattice Thermal conductivity:-}

For simplicity, the subscript (q) and (s) will be omitted. In the bulk the solution for equation (9) can be written as:-

$\widetilde{N}=\frac{\partial N^{\circ}}{\partial T} \nabla T . V \tau_{C}$

In the coordinate system that we have defined, $\tilde{N}$ can further written as:$\widetilde{N}=\frac{\partial N^{\circ}}{\partial T} \frac{\partial T}{\partial z} V \tau_{C}$

Substituting eq(3) and eq(11) in eq(1) and comparing the result with eq.(3), a regular bulk formulais can be obtained for the lattice thermal conductivity:-

$k=\left(\frac{K_{B}}{\hbar}\right)^{3}\left(\frac{K_{B}}{2 \pi^{2} v_{g}}\right) T^{3} \int_{0}^{\frac{\theta_{D}}{T}} \frac{\tau_{C} X^{4} e^{x}}{\left(e^{x}-1\right)^{2}} d x$

This equation is Klemens-Galloway's expression for the lattice thermal conductivity in bulk material, where $x=\hbar \omega / K_{B} T, K_{B}$ is the Boltzmann constant, $\hbar$ is the Dirac constant, $\tau_{\mathrm{c}}$ is the combined relaxation time, and $\mathrm{v}_{\mathrm{g}}$ is the phonon group velocity, $\theta_{\mathrm{D}}$ is the Deby temperature (Callaway., 1958). For nanowires and of quantum well Deby temperature $\theta_{\mathrm{D}}$ may be determined by the equation:-

$$
\frac{v_{D}}{v_{b}}=\frac{\theta_{D}}{\theta_{b}}
$$

Where $v_{D}$ and $v_{b}$ are group velocities of nanowires (or quantum well) and bulk respectively, and $\theta_{\mathrm{b}}$ represent Deby temperature of silicon bulk (Liang et al ., 2006). Callaway considered this phenomenological model in which he treats the speed of sound as a constant (no difference between group velocity and phase velocity) similar to that, in the Deby theory of the specific heat. His calculation contained acoustic branches, while the contribution of the optical modes neglected. This equation $(\mathrm{eq}(12))$ has been used with an appropriate Modification of the phonon 
group velocity and combined relaxation time in a quantum well and nanowires, because phonons have different velocities and different energy .Eq.(12), is valid under assumption that the resistance scattering mechanisms, which do not conserve crystal momentum, are dominant. These are the processes that contribute to the lattice thermal resistance (Khitun et al ., 1999; Zou et al 2001).

\section{3- Phonon dispersion and group velocities:-}

The acoustic phonon dispersion in free standing nanowires (or quantum well) calculated by considering only the in-plane transport in the well and the main contributions in the plane heat transfer comes from the longitudinal acoustic phonon modes. (Zou et al 2001)

The dispersion relation for confined phonons in a nanowires of diameter $\mathrm{D}$ is given by :-

$$
\left(q^{2}-q_{t}^{2}\right)^{2} \frac{\left(q_{d} D / 2\right) J_{\circ}\left(q_{d} D / 2\right)}{J_{1}\left(q_{d} D / 2\right)}-2 q_{d}^{2}\left(q^{2}+q_{t}^{2}\right)+4 q^{2} q_{d}^{2} \frac{\left(q_{t} D / 2\right) J_{\circ}\left(q_{t} D / 2\right)}{J_{1}\left(q_{t} D / 2\right)}=0
$$

The dispersion relation for quantum well of thickness D can be written as:-

$$
\frac{\tan \left(q_{t} D / 2\right)}{\tan \left(q_{d} D / 2\right)}=-\frac{4 q^{3} q_{t} q_{d}}{\left(q^{2}-q_{t}^{2}\right)^{2}}
$$

Where $\mathrm{q}_{\mathrm{t}}, \mathrm{q}_{\mathrm{d}}$ are longitudinal and transverse phonon wave vectors, $\mathrm{J}_{\mathrm{o}}$ and $\mathrm{J}_{1}$ are the ordinary Bessel functions, and $\mathrm{q}_{\mathrm{t}}$ and $\mathrm{q}_{\mathrm{d}}$ are two parameters related by :-

$$
q_{d, t}^{2}=\frac{\omega^{2}}{v_{d, t}^{2}}-q^{2}
$$

Here, $v_{d}=\sqrt{ }(\lambda+2 \mu) / \rho$ and $v_{t}=\sqrt{ } \mu / \rho$ are the longitudinal and transverse sound velocity in the bulk material respectively, and $\lambda, \mu$, are lame constant and $\rho$ is the density (Khitun et al., 1999; Zou et al 2001). The phonon dispersion relation can be written as:-

$$
\omega_{n}=v_{d, t}\left(q^{2}+q_{d, t n}^{2}\right)^{1 / 2}
$$

Where $\omega_{\mathrm{n}}$ is the phonon frequency for the nth branch. Confined phonon dispersion is obtained by numerically solving both equation(14) (or equation(15)) and equation(16) at each $\mathrm{q}$, there are many solutions for $\mathrm{q}_{\mathrm{d}}$ and $\mathrm{q}_{\mathrm{t}}$. phonon group velocity is calculated for each branch by numerical differentiation. Phonon group velocity for each branch is:-

$$
v_{n}=\frac{d \omega_{n}}{d q}
$$

Since different branches have different group velocities, the phonon group velocity averaged overall contributing branch calculated by using the population averaged phonon group velocity: 


$$
V^{\prime}(\hbar \omega) \cong \sum_{n} v_{g, n}(\hbar \omega) \frac{N_{n}(\omega)}{\sum_{n} N_{n}(\omega)}=\sum_{n} v_{g, n}(\hbar \omega) \frac{e^{\left(n \frac{\hbar \omega}{k_{B} T}\right)}}{\sum_{n} e^{\left(n \frac{\hbar \omega}{k_{B} T}\right)}}
$$

Where, $\mathrm{v}_{\mathrm{g}, \mathrm{n}}$ is the group velocity of the $\mathrm{nth}$ mode, the population group velocity is then used to calculate the lattice thermal conductivity. (Khitun et al ., 1999; Zou et al 2001)

\section{4-phonon relaxation times:-}

The combined phonon relaxation time is obtained from the Mathiessens rule:-:

$$
\frac{1}{\tau_{C}}=\frac{1}{\tau_{u}}+\frac{1}{\tau_{B}}+\frac{1}{\tau_{M}}
$$

Here, $\tau_{\mathrm{u}}$ is the three-phonon umklapp scattering due to anharmonic nature of the crystal potential energy, $\tau_{\mathrm{M}}$ is the mass- difference scattering of phonon and $\tau_{\mathrm{B}}$ are relaxation times due to boundary scattering (Khitun et al., 1999; Balandin et al., 1998). Phonon spatial confinement leads to modification of the phonon dispersion and phonon group velocity, and hence to a change of phonon scattering rate.

The relaxation time for Umklapp scattering is given by Klemens as:-

$$
\frac{1}{\tau_{u}}=2 \gamma^{2}\left(\frac{K_{B} T}{\mu V_{\circ}}\right)\left(\frac{\omega^{2}}{\omega_{D}}\right)
$$

Where $\gamma$ is the Gruneisen anharmonicity parameter, $\mu$ is the shear modulus $\mathrm{V}_{0}$ is the volume per atom, and $\omega_{\mathrm{D}}$ is the Deby frequency.

Mass- difference scattering (is also-called isotope scattering) is the scattering of phonons due to the presence of atoms with a mass different from the average atomic mass in a semiconductor. The relaxation rate for the mass-difference scattering is calculated using the following expression:-

$$
\frac{1}{\tau_{M}}=\left(\frac{V_{0} \omega^{4} \Gamma_{m}}{4 \pi v_{g}^{3}}\right)
$$

Here $\mathrm{V}_{0}$ is the volume per atom, $\omega$ is the phonon frequency, and $\Gamma \mathrm{m}$ is the measure of the strength of the mass-difference scattering defined as:$\Gamma_{\mathrm{m}}=\Sigma \mathrm{fi}\left[\left(\Delta \mathrm{M}_{\mathrm{i}} / \mathrm{M}^{\prime}\right)^{2}\right]$

Where, fi is the fractional content of mass $\mathrm{M}_{\mathrm{i}}$, which is different from the mass $\mathrm{M}$ of the main atom, and $\Delta \mathrm{M}_{\mathrm{i}}=\mathrm{M}-\mathrm{M}_{\mathrm{i}}$, and $\mathrm{M}^{\top}$ is given by: $\mathrm{M}^{\prime}=\Sigma \mathrm{f}_{\mathrm{i}} \mathrm{M}_{\mathrm{i}}$, is the average atomic mass. Since in nature silicon always contains a mixture of three main isotopes $\left(92 \%\right.$ of $\mathrm{Si}^{28}, 4.9 \%$ of $\mathrm{Si}^{29}$, and $3.1 \%$ of $\mathrm{Si}^{30}$ ) the isotope scattering significantly contributes to the thermal resistance of the material ((Khitun et al., 1999; Balandin et al., 1998). 
Boundary scattering rate can be calculated by using modified boundary scattering:-

$$
\frac{1}{\tau_{B}}=\frac{v_{g}}{D}(1-p)
$$

Here, $\mathbf{p}$ is the parameter which is related to the interface roughens. The value of $\mathbf{p}$ represent the probability that the phonon is undergoing a specular scattering event at the interface (Zou et al., 2001), where $\mathbf{p}=1$, (purely specular scattering) boundary scattering does not contribute to the thermal resistance. In the case of purely diffuse scattering, the above expressions reduce to:-

$$
\frac{1}{\tau_{B}}=\frac{v_{g}}{D}
$$

This is the well known formula in the Casimer limit (Casimer., 1938).

\section{Results and Discussion}

Size effect (boundary scattering) and spatial confinement of phonon causes change in the dispersion relation, such changes can modify the group velocity, this is due to the increase of the scattering rate and all these effects suppressed thermal conductivity. From the following, all these results are due to the reduce of the size of material:-

\section{1- dispersion relation for quantum well and Nanowires:-}

First, using numerical method (Muller-Secant method), (MathCAD) software can be used for solving both eq (14) and eq(15), the material parameter used in the numerical solution was:-

$\mathrm{v}_{\mathrm{d}}=8.478 \times 10^{5} \mathrm{~cm} / \mathrm{sec}$ and $\mathrm{v}_{\mathrm{t}}=5.846 \times 10^{5} \mathrm{~cm} / \mathrm{sec}$. Figure(1) and figure (2) shows the acoustic phonon dispersion for a free-standing silicon cylindrical nanowires of diameter $\mathrm{D}=10-\mathrm{nm}$ and quantum well of the same size. It is easy to see that there are more dispersion and velocity branches for each polarization type as compared to the bulk,

The first branches in both structures are of the true acoustic type, i.e that only the first branch has a linear dispersion relation, in this case $\mathrm{q}=0$ gives $\mathrm{w}=0$. For the higher mode numbers there exists a cut-off frequency (i.e $\mathrm{w} \neq 0$ when $\mathrm{q}=0$ ) for both the nanowires and quantum well.

The cut-off frequency for second branch in the nanowires is larger than that in a quantum well (as shown in figure (1) and figure (2)) and this is due to tighter phonon spatial confined in nanowires than that in a quantum well. (Alassafee.,2005; Zou et al 2001)

\section{2- Group velocity:-}

The phonon dispersion relation change will lead to the reduction of phonon group velocity, the slopes of the phonon dispersion branches 
represent the group velocity $\left(\mathrm{v}_{\mathrm{g}}{ }^{(\mathrm{n})}=\partial \mathrm{w}_{\mathrm{n}} / \partial \mathrm{q}\right)$. It is easy to see that there are more dispersion and velocity branches for each mode as compared to the bulk, and group velocities of all branches decrease. The higher the modes number the smaller the group velocity.

By using numerical differentiation, the exact value of the group velocity for each phonon branch is determined. Figure (3) and figure (4) shows the phonon group velocity as a function of the phonon energy of well and wire, the over all value of the population averaged phonon group velocity of quantum well is $\left(5.56 \times 10^{3} \mathrm{~m} / \mathrm{sec}\right)$, and for nanowires is $\left(5.45 \times 10^{3} \mathrm{~m} / \mathrm{sec}\right)$. In bulk silicon, the longitudinal sound velocity is $\left(8.4 \times 10^{3} \mathrm{~m} / \mathrm{sec}\right)$.

The important consequence from this is that the effective phonon group velocity will depend on the size of the material structures. A large reduction occurs in the nanowires than that in the well due to tighter phonon confinement. (Zou et al., 2001; Chen et al., 2005). For small values of the phonon energy the group velocity for the first branch almost coincide with the bulk velocity.

\section{3- Phonon relaxation rate:-}

Confinement of acoustic phonons and corresponding change in their group velocities lead to an increase in the phonon relaxation rate. Combined phonon relaxation rates calculated by using equation (19). Following material parameter has been used in the calculation:$\gamma=0.56$, and density $\rho=2.329 \times 10^{3} \quad\left(\mathrm{~kg} / \mathrm{m}^{3}\right)$, the shear modules $\mu=7.96 \times 10^{10}\left(\mathrm{~kg} / \mathrm{sec}^{2} . \mathrm{m}\right)$, volume of

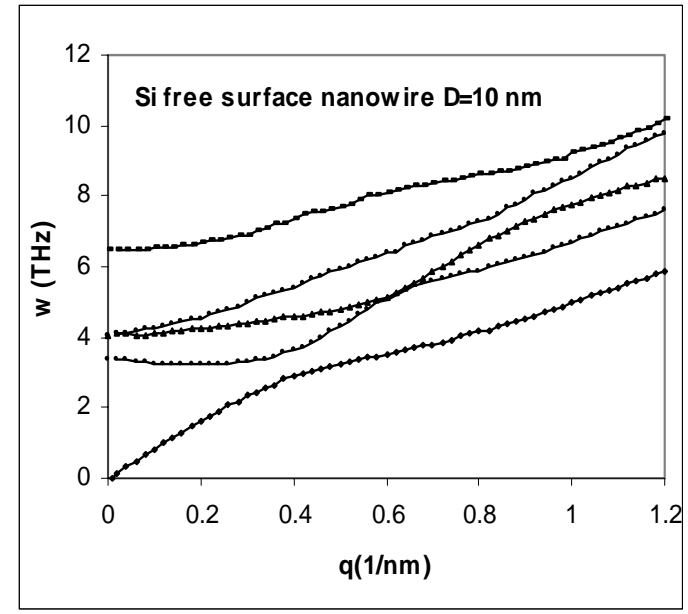

Figure (1): Acoustic phonon dispersion for long-. itudinal modes in silicon nanowires of $D=10 \mathrm{~nm}$

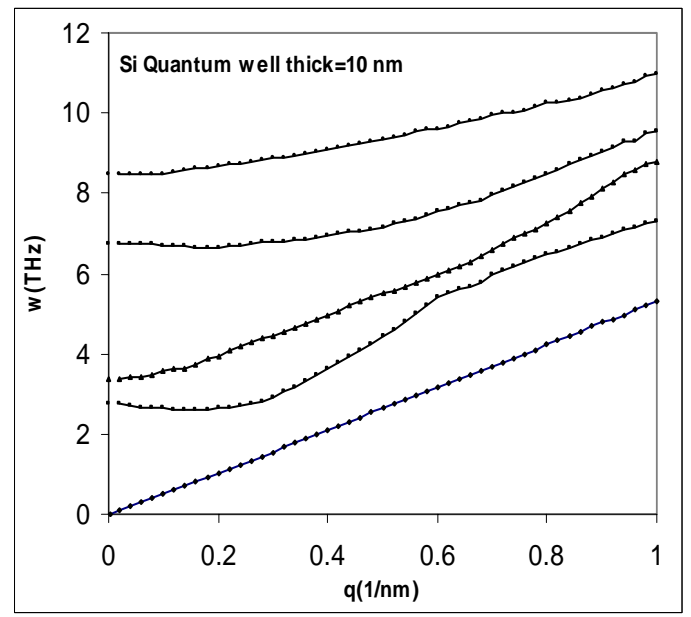

Figure (2): Acoustic phonon modes in silicon quantum well with thickness is $10-\mathrm{nm}$ 


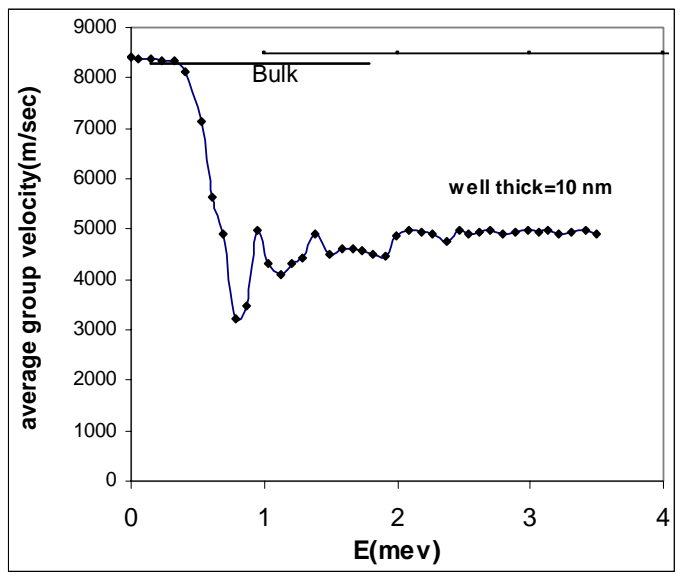

Figure (3): Average phonon group velocity as a function of phonon energy for Si quantum well, the well thickness is $10-n m$.

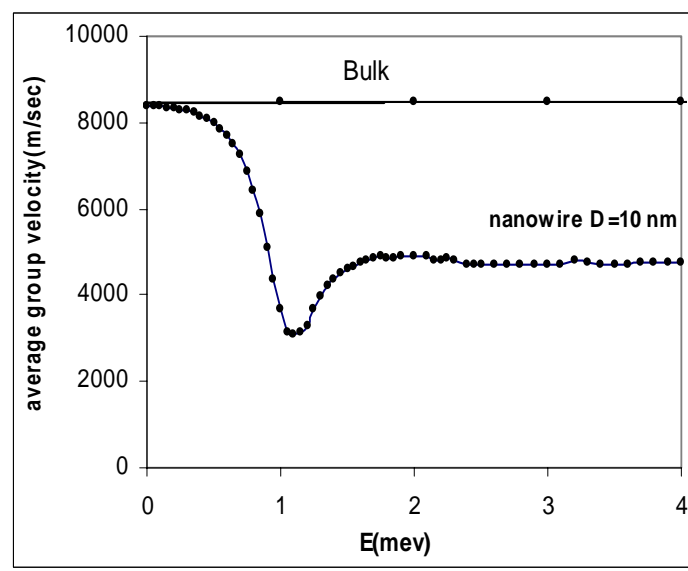

Figure (4): Average phonon group velocity as a function of phonon energy for Si nanowires, the wire Diameter is $10 \mathrm{~nm}$.

silicon atom $\mathrm{V}_{\mathrm{o}}=2.002 \times 10^{-29} \mathrm{~m}^{3}$, isotopic factor $\Gamma=2.5 \times 10^{-4}$. (Alassafee., 2005; McConnell et al., 2005).

Cut- off frequency calculated by using the following equation:-

$$
\omega_{c}=\frac{2 \kappa_{B} \theta_{D}}{\pi \hbar}
$$

Where $\theta_{\mathrm{D}}$ is the Deby temperature (Liang et al., 2006), for silicon nanowires it is equal to $(403 \mathrm{~K})$, and equal to $(410 \mathrm{~K})$ for quantum well, corresponding to the cut-off frequencies $\mathrm{wc}=3.356 \times 10^{13}(1 / \mathrm{sec})$ of nanowires and $\mathrm{wc}=3.41 \times 10^{13}(1 / \mathrm{sec})$ of quantum well.

\section{3-A- Umklapp and Mass-difference scattering:-}

Umklapp scattering is related to the resistive phonon-phonon scattering and it is due to the anharmonic nature of the crystal potential energy (three phonon Umklapp scattering) (Chen et al., 2005). Figure (5a) shows that umklapp scattering in the nanowires and it is greater than quantum well over most of the large portion of the frequency range and it is due to stronger spatial confinement of phonon in the nanowires

Mass-difference shown in figure (5-b), these scattering arise from the presence of atoms with different mass, this scattering is also affected by spatial confinement of phonon through the group velocity (Balandin et al 1998). From the figure (5-b) Mass-difference scattering in the nanowires is larger than quantum well.

\section{3-B:- Boundary scattering}

For the quantum well and wire having a diameter of $\mathrm{D} \leq 30-\mathrm{nm}$, the boundary scattering will dominates over all-scattering process Figure (6a-b) shows the relaxation rates due to different scattering mechanisms as a function of phonon frequency for nanowires and quantum well. 
In this figure also show that the boundary scattering dominate overall scattering rate in both nanowires and well, and this is due to the long wave length of phonon comparable with the dimension of the sample.

In bulk materials, this boundary scattering regime can be approached only at low temperatures, that's when the phonon mean free path becomes comparable to the sample size. In the bulk, Umklapp scattering is dominates over the mass-difference as shown in figure(7), the same is true for quantum well and nanowires (figure (6-a-b)). (Zou. J, Balandin. A., (2000) Alassafee., 2005)

From both figure(6) and figure(7) we observed the increase of scattering in wire and well comparable with bulk, Umklapp scattering in nanowires is (2.3) times larger than bulk and in well (1.7) times larger than bulk and mass-difference scattering in wire and well are one order larger than that of bulk, these are because of the modification of the phonon dispersion due to spatial confinement of the phonon modes. (Zou et al., 2000; Alassafee., 2005).

\section{4- Calculation of lattice thermal conductivity:-}

After group velocity and relaxation rate are found, the lattice thermal conductivity in the temperature range of $2-300 \mathrm{~K}$ are calculated by using equation (12).

Figure( $8-a-b)$ shows the effect of the Umklapp, Mass-Difference and boundary scattering on the lattice thermal conductivity in the nanowires and quantum well. For Umklapp scattering, the lattice thermal conductivity in silicon nanowires at $300 \mathrm{~K}$ is reduced to about $60 \%$ of its bulk value, i.e. Umklapp scattering cause to drop the lattice thermal conductivity to about 1.67 times of the bulk (Figure -9). In the quantum well umklapp scattering reduced to $62 \%$ of its bulk value and thermal
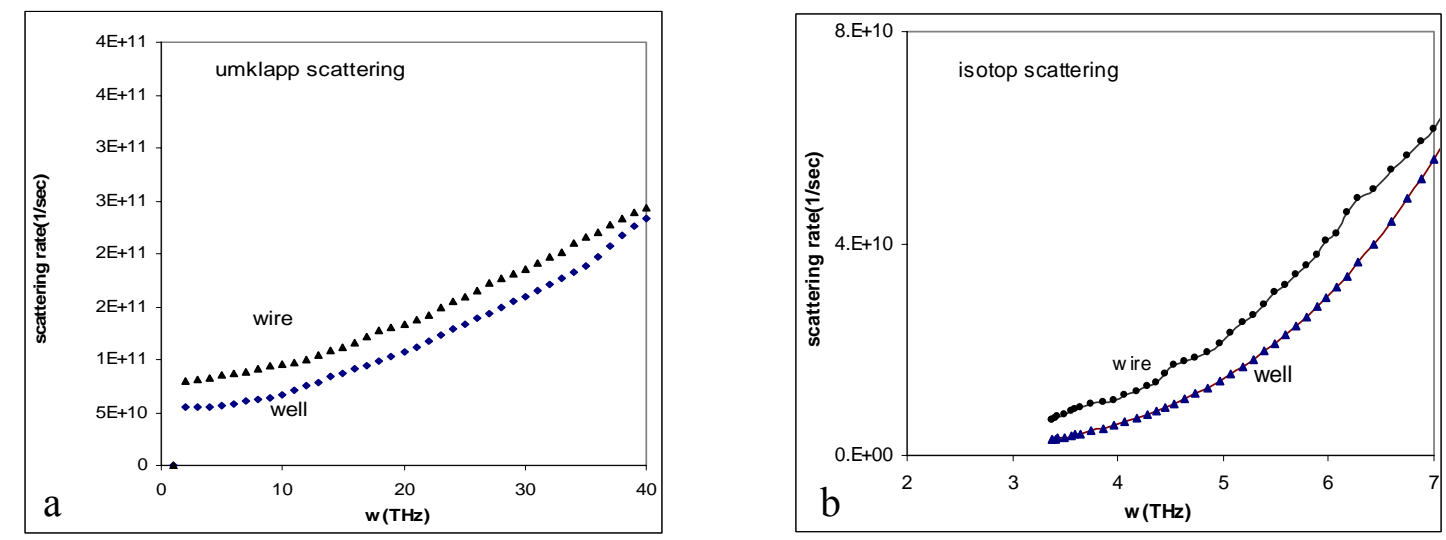

Figure(5). (a): shows the scattering rate for Umklapp scattering, and (b): Mass- difference scattering rate both as a function of frequency at $300 \mathrm{~K}$ for nanowires and quantum well. 

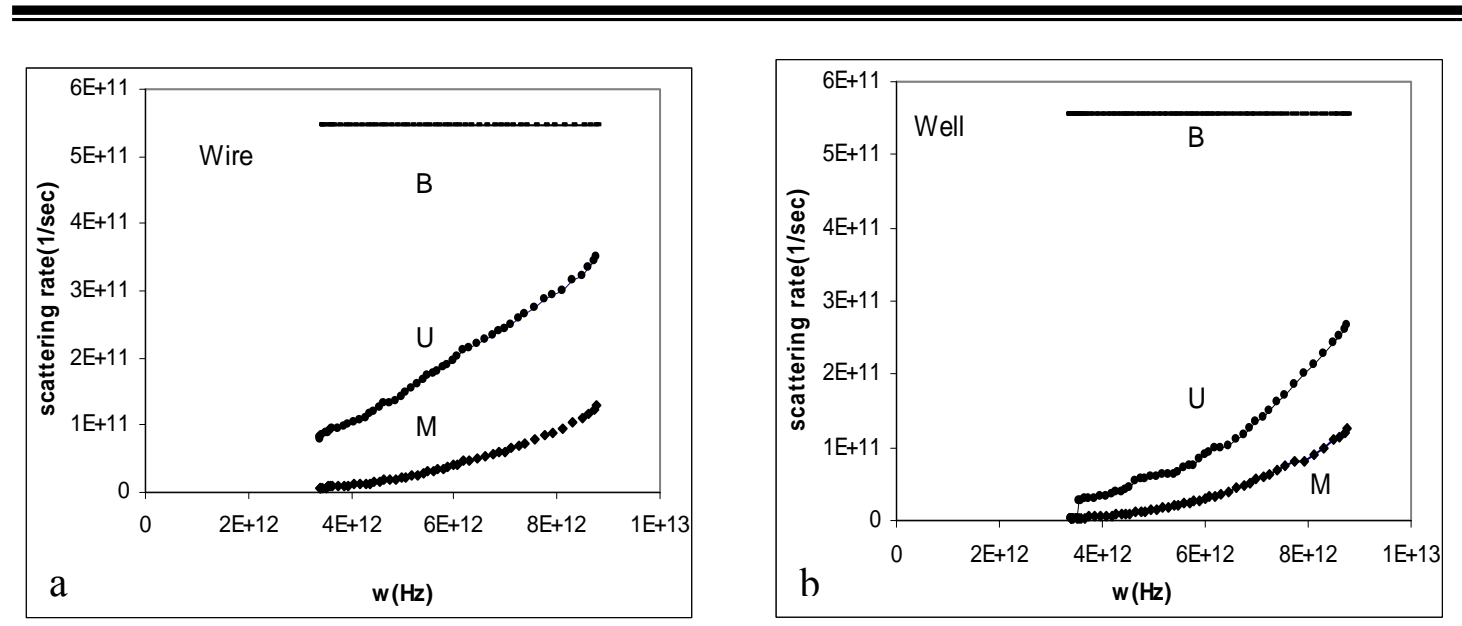

Figure (6): phonon scattering rates in silicon (a): nanowires and (b): quantum well due to Umklapp(U), Mass-difference (M) and Boundary scattering (b) as a function of frequency at $300 \mathrm{~K}$.

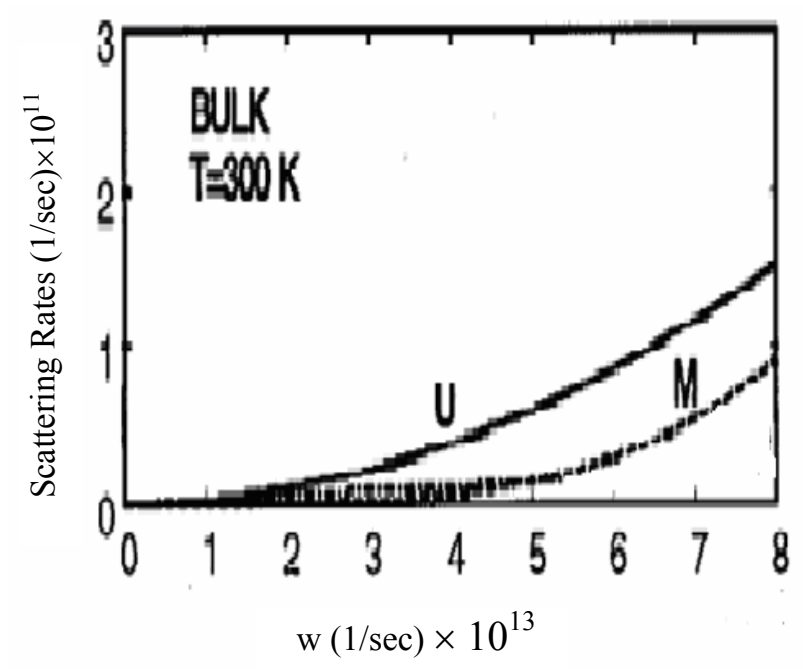

Figure(7): Phonon scattering rates due to umklapp and mass-difference scattering in silicon bulk material as a function of frequency at 300K.( Zou. J, Balandin. A,(2000))

conductivity drop 1.61 times of the bulk value at $300 \mathrm{~K}$ (Figure -9) Mass-Difference scattering cause thermal conductivity in nanowires to reduced to about $82 \%$ of its bulk value, and in quantum well lattice thermal conductivity reduced to about $83 \%$ of the bulk value.

The boundary effect in the nanoscale size occurs at room temperature and because at this temperature the well and wires thickness becomes smaller than the room temperature phonon mean free path (Khitun et al 1999). At room temperature lattice thermal conductivity of the bulk is equal to 148 (W/m.K) (McConnell et al 2005). From our calculation the value of lattice thermal conductivity of nanowires of diameter $\mathrm{D}=10-\mathrm{nm}$ at room temperature is equal to $9(\mathrm{~W} / \mathrm{m} . \mathrm{K})$, and for quantum well Lattice thermal conductivity of thickness $10-\mathrm{nm}$ equal to $9.3(\mathrm{~W} / \mathrm{m} . \mathrm{K})$. The over all thermal conductivity of nanowires at $300 \mathrm{~K}$ is about $6.1 \%$ of the bulk silicon, and for quantum well about $6.3 \%$ of the bulk value. 
Keiven. Etessam, and M. Ashegi, (2005). measured thermal conductivity of silicon thin films for different film thickness $(100,50,25$, $10-\mathrm{nm})$ as shown in figure (10). Balandin et al, calculated thermal conductivity of quantum well for the same size they proved that thermal conductivity reduces to about $13 \%$ of the bulk value (Balandin. A, Wang. K.L,(1998)).

The experimental value of lattice thermal conductivity of nanowires of diameter 10-nm is equal to 6.1(W/m.K) (Alassafee., 2005).

Yunifei Chen, Deyu Li and others using Monte Carlo Simulation (Figure-11) to calculate lattice thermal conductivity of nanowires of diameter $\mathrm{D}=10 \mathrm{~nm}$ at $300 \mathrm{~K}$ and they show that it is equal to $10(\mathrm{~W} / \mathrm{m} . \mathrm{K})$ (Chen et al., 2005). Table (1) shows the value of thermal conductivity of silicon quantum well for different thickness and the percent reduction of lattice thermal conductivities comparable with silicon bulk value (Alassafee., 2007; McConnell et al ., 2005)

\begin{tabular}{|l|l|}
\hline Quantum well thickness & Percent reduced \\
\hline $1 \mu \mathrm{m}$ & $10 \%$ \\
\hline $100 \mathrm{~nm}$ & $50 \%$ \\
\hline $50 \mathrm{~nm}$ & $70 \%$ \\
\hline $20 \mathrm{~nm}$ & $82 \%$ \\
\hline $10 \mathrm{~nm} *$ & $94 \%$ \\
\hline *present work &
\end{tabular}

Tab.(1): silicon quantum well thickness and the percent reduced thermal conductivity value at $300 \mathrm{~K}$.

\section{Conclusion}

In this paper, Lattice thermal conductivity of silicon nanowires and quantum well of order $10-\mathrm{nm}$ calculated in room temperature by considering the effect of confinements of phonons on the dispersion relation and the group velocity on the lattice thermal conductivity. Analysis showed that
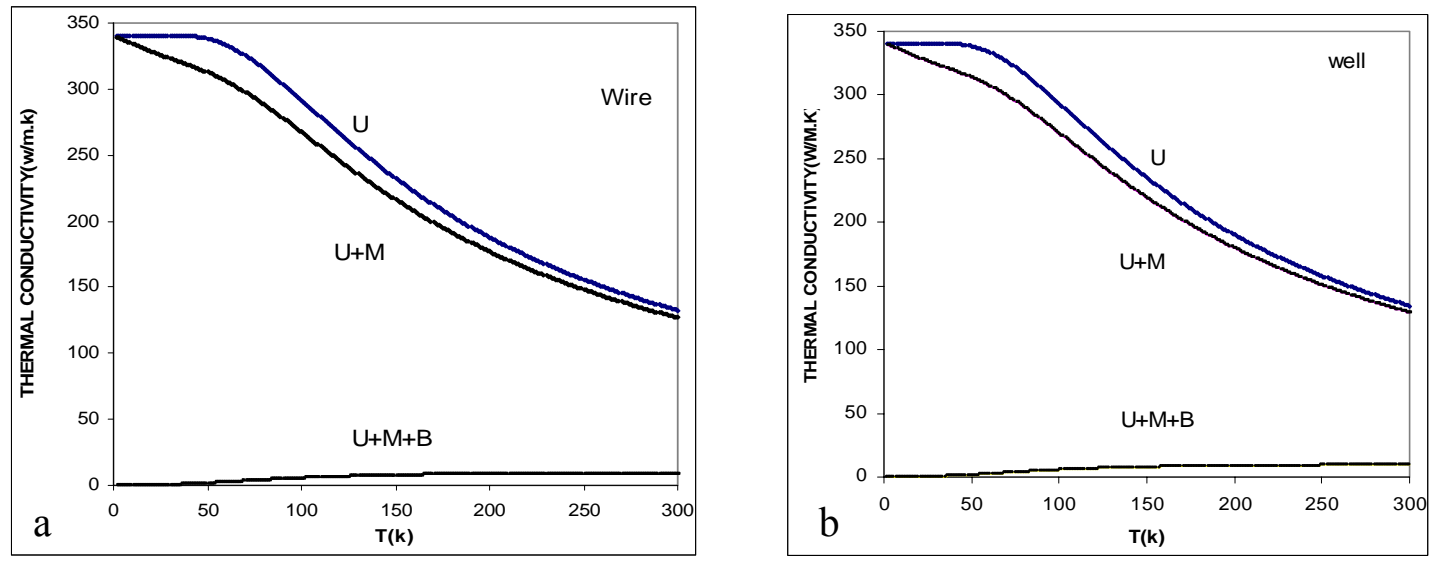

Figure(8): Lattice thermal conductivity as a function of temperature for:-

(a) silicon nanowires of diameter $D=10 \mathrm{~nm}$, and

(b) silicon quantum well of thickness $=10 \mathrm{~nm}$. 


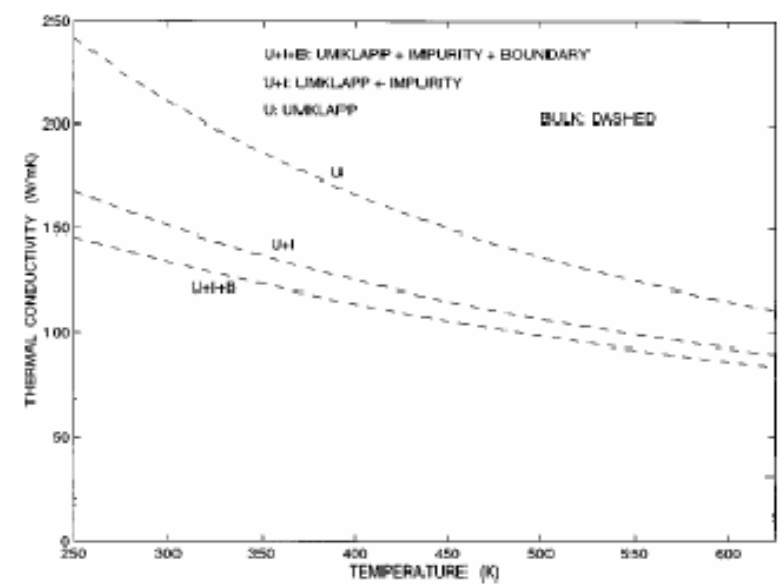

Figure(9): Lattice thermal conductivity as a function of temperature for silicon bulk material.

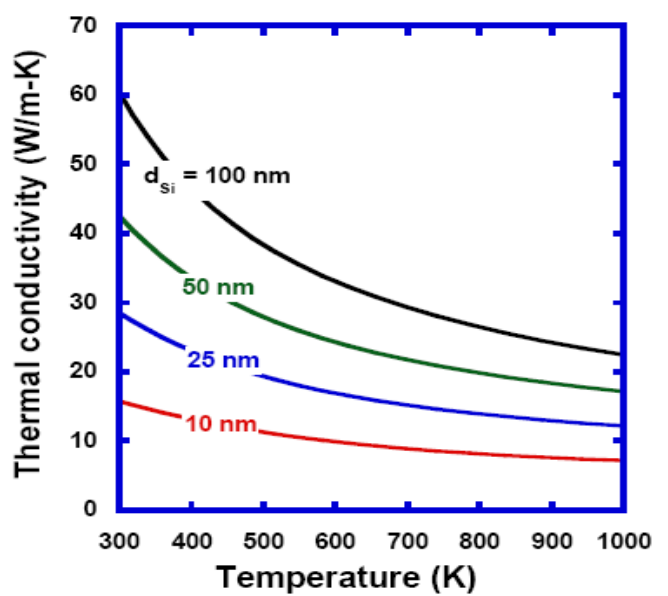

Figure(10). Measured thermal conductivity as a function of temperature of silicon thin films for different film thickness(Keiven., Ashegi , 2005).

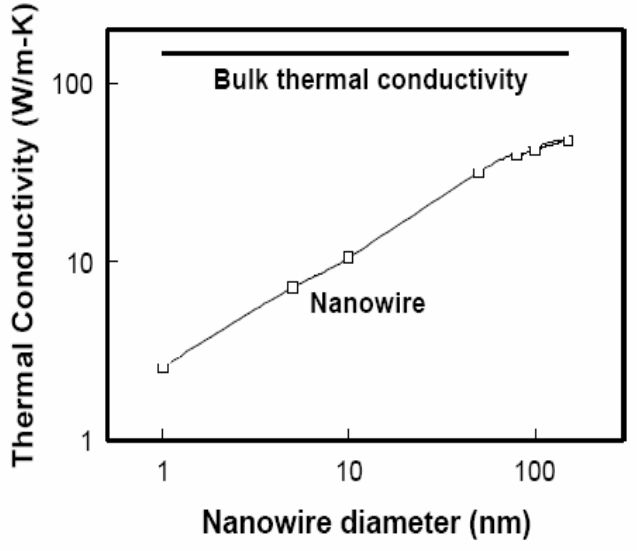

Figure(11): Nanowires thermal conductivity versus diameter at $\mathrm{T}=300 \mathrm{k}(\mathrm{Chen}$. $Y$, Li.D, Lukes . J. R, Majumdar. A, (2005)

the scattering rates by confined acoustic phonons exceed corresponding to the bulk. Size effect lead to the value of thermal conductivity differs from that of the bulk, this is due to the fact that the boundary scattering dominated over all scattering process in the nano range, and results show the good agreement between the experimental data and theoretical calculation. 


\section{References}

1) Alassafee. A, (2005), Lattice Thermal Conductivity of Silicon Nanowires M.Sc. Thesis, University of Salahaddin.

2) Balandin. A, Wang. K.L, (1998), Significant decrease of the Lattice Thermal Conductivity due to Phonon Confinement in a Freestanding Semiconductor Quantum Well, Physical Review B. 58,3 1546.

3) Callawy. J, (1958). Model for Lattice Thermal Conductivity at Low Temperature, Phys. Rev. 113, 4.1046.

4) Casimir. H, (1938). Note on the Conduction of heat in Crystal, Physica (Utr). 6,495.

5) Chen. Y, Li. D, Lukes. J. R, Majumdar. A, (2005), Monte Carlo Simulation of Silicon Nanowire Thermal Conductivity, Journal of Heat Transfer. 127/1129

6) Feng. X. L, Li. Z. X., Z, Gue. Z. Y, (2003), Molecular Dynamics Simulation of Thermal Conductivity of Nanoscale Thin Films, Microescale Thermo physical Engineering, 7:153-161.

7) Khitun. A, Balandin. A, Wang. K. L, (1999). Modificatio of the Lattice Thermal Conductivity in Silicon Quantum Wires Due to Spatial Confinment of Acoustic Phonons, Supperlattice and Microstructures 0772,29. 3 p 181-190.

8) Keiven. Etessam, Mehdi Ashegi, (2005). Impact of thermal subcontinuum effects on electrical performance of silicon -on- insulator transistor. The ASME/ Pacific rim technology conference and exhibition on integration and packing of MEMS, NEMS, and electronic systems.

9) Liang. L. H, Baown, L, (2006), Size- Dependent Thermal Conductivity of Nanoscale Semiconducting systems, Nanjiang, Singapor. 210093-117597.

10) Mukdadi M. O, Datta. S. K., Dunn. M, L, (2005), Acoustic-phonon dispersion in nanowires, Journal of applied physics 97, 074313-1

11) McConnell. A. D, Goodson K. E, (2005), Thermal Conduction Silicon Micro- AND Nanostructures. Annual review of Heat Transfer, .14.129

12) Alassafee. A, (2007), phonon heat conduction in silicon films with thickness of order $20 \mathrm{~nm}$ (un published).

13) Yang. C. C, Xiao. M. X, Li. W., Jiang. Q, (2006), Size effect on Deby temperature, Einestein temperature, and Volume thermal expansion coefficient of Nanocrystals, Solid State Communications, $139,148-152$.

14) Zou. J, Balandin. A, (2000), Phonon Heat Conduction in a Semiconductor Nanowires, Journal of applied physics, 89, 5, 2933 89, 5, 2933.

15) Zou. J, Balandin .A, (2001). Development of an Ab-initio Model of the Laatice Thermal Conductivity in Semiconductor Thin Films and Nanowires, University of Califronia, 9252. U S.A. 